\title{
Diagnosis and surgical resection of solitary pulmonary nodules in patients with breast cancer
}

\author{
KAZUMI TANAKA $^{1}$, KIMIHIRO SHIMIZU ${ }^{1}$, YOICHI OHTAKI ${ }^{1}$, TETSUHIRO NAKANO ${ }^{1}$, \\ MITSUHIRO KAMIYOSHIHARA ${ }^{1}$, KYOICHI KAIRA ${ }^{2}$, NANA ROKUTANDA ${ }^{1}$, \\ JUN HORIGUCHI $^{1}$, TETSUNARI OYAMA ${ }^{3}$ and IZUMI TAKEYOSHI ${ }^{1}$ \\ ${ }^{1}$ Division of Thoracic and Visceral Organ Surgery; ${ }^{2}$ Department of Medicine and Molecular Science, \\ and ${ }^{3}$ Division of Diagnostic Pathology, Graduate School of Medicine, Gunma University, \\ Maebashi, Gunma 371-8511, Japan
}

Received June 8, 2012; Accepted August 2, 2012

DOI: $10.3892 / \mathrm{mco} .2012 .21$

\begin{abstract}
The differential diagnosis of solitary pulmonary nodules (SPNs) using radiological features in patients with breast cancer is vital yet difficult. The nature of SPNs, of these patients, as well as the role of surgery in the diagnosis and treatment were evaluated. Thirty consecutive patients, who underwent surgery for an SPN between 2002 and 2011 subsequent to curative surgery for breast cancer were retrospectively evaluated. The overall survival and disease-free survival periods were estimated using the Kaplan-Meier method. The Student's t-test and the $\chi^{2}$ test were performed to compare percentages and the mean values, respectively. The values were considered statistically significant at $\mathrm{P}<0.05$. Most (93\%) SPNs were malignant. The pathological diagnoses in patients with SPNs were primary lung cancer $(\mathrm{n}=20$, $67 \%)$, pulmonary metastasis from breast $(\mathrm{n}=7,23 \%)$ or colon $(\mathrm{n}=1,3 \%)$ cancers and benign conditions $(\mathrm{n}=2,7 \%)$. Of the 20 patients with primary lung cancer, $15(75 \%)$ had stage IA tumors (T1aNOM0). The average disease-free interval was significantly longer in patients with primary lung cancer compared to patients with pulmonary metastases from breast cancer $(\mathrm{P}=0.031)$. The five-year survival rates after pulmonary resection for lung metastasis from breast cancer patients and primary lung cancer patients were 100 and $61.1 \%$, respectively. SPNs found in patients with breast cancer were found to have a high probability of malignancy, especially primary lung adenocarcinoma. The early resection of SPNs in patients diagnosed with both primary and metastatic lung cancer led to a good prognosis. The early pathological diagnosis by
\end{abstract}

Correspondence to: Dr Kimihiro Shimizu, Division of Thoracic and Visceral Organ Surgery, Graduate School of Medicine, Gunma University, 3-39-22 Showa-machi, Maebashi, Gunma 371-8511, Japan E-mail:kmshimizu@gmail.com

Key words: solitary pulmonary nodule, primary lung cancer, breast cancer, surgery, metastasectomy, video-assisted thoracoscopic surgery surgical resection was suggested to be conducted for the early diagnosis and appropriate treatment of SPNs in patients with breast cancer.

\section{Introduction}

In women, lung cancer is the most common cause of cancer-related mortality and the second most commonly occurring cancer worldwide (1). Lung cancer is directly associated with a delayed presentation. Signs and symptoms are rarely present until the malignancy is advanced and often unresectable. Patients with stage IA (T1NOM0) disease have a 61-75\% five-year survival rate subsequent to surgical resection (2). Most of these patients have no symptoms and their lung lesions are initially detected as solitary pulmonary nodules (SPNs) in computed tomography (CT) screening.

An SPN is defined as a focal round or oval area (diameter $<3 \mathrm{~cm}$ ) of increased radiographic opacity in the lung (3). In the United States, $>150,000$ patients visit their physicians annually with symptoms generating a diagnostic dilemma of an SPN. CT screening has increased the detection rate of SPNs with its detection rate increasing even further, due to incidental findings of lung nodules on chest CT (2).

In the past 30 years, substantial advances have been made in the outcome of patients with early-stage breast cancer, resulting from early detection, the identification of prognostic factors and the improvements in surgical techniques, such as radio- and systemic therapy (4). These improvements in breast cancer outcomes have increased the curability or survival period of several patients, thereby increasing their risk of developing a second malignancy.

Pulmonary metastases are common in patients with breast cancer. Most physicians traditionally consider an SPN occurring in a patient with breast cancer to represent a metastasis from the breast (5). However, a differential diagnosis using radiological features is often difficult $(5,6)$. Thus, an SPN occurring in a patient with documented past or present breast cancer presents a diagnostic challenge.

The nature of SPNs in patients with breast cancer and the effectiveness of surgery in their treatment were retrospectively evaluated. 


\section{Materials and methods}

Patient population and surgical procedure. In total, 30 SPN specimens were obtained from women (mean age, 58 years; range, 41-80 years), who underwent surgical resection at the Gunma University Hospital between January, 2002 and July, 2011. Institutional approval and written informed consent from the patients were obtained prior to surgery. The patients had previously undergone curative surgery for breast cancer. The patient characteristics are shown in Table I. Patients with metastases in other organs were excluded. Pre-operative evaluation included chest X-rays, total body CT scans, fiberoptic bronchoscopy, isotopic bone scanning, cardiopulmonary function tests and total body fluorine-18 deoxyglucose positron emission tomography (FDG-PET). Peripheral lesions were submitted pre-operatively to CT-guided needle aspiration biopsy (CT-NAB), where the lesion diameter and location were appropriate. No patient had a centrally located SPN and no transbronchial needle aspiration or other bronchoscopic sampling procedure was required during pre-operative bronchoscopy.

Wedge resection or segmentectomy by video-assisted thoracoscopic surgery (VATS) was planned to confirm the diagnosis of peripherally-located SPNs. Frozen sections with hematoxylin and eosin (H\&E) staining were evaluated intraoperatively and the operation was terminated when a nodule was definitively diagnosed as metastatic lung cancer or a benign condition. In cases of nodules diagnosed as primary lung cancer or suspected as malignant, although not identified as either primary or metastatic lung cancer at intraoperative diagnosis, the VATS was converted to an open procedure including anatomical resection (lobular dissection or segmentectomy) and hilar and mediastinal lymph node dissections.

Pathological examination and immunohistochemical diagnosis. For a strict differential diagnosis of pulmonary nodules, a pathological examination was performed by two or more pathologists. For the histological diagnosis, a conventional morphopathological examination using $\mathrm{H} \& \mathrm{E}$ staining was performed. The pathological findings were evaluated and compared to those of primary breast cancer. For the strict differential diagnosis of pulmonary nodules, immunohistochemical examination was performed using the standard avidin-biotin peroxidase complex method, with monoclonal antibodies against estrogen receptor (ER)- $\alpha$, progesterone receptor (PR), gross cystic disease fluid protein-15 (GCDFP-15), thyroid transcription factor-1 (TTF-1) and surfactant apoprotein-A (SP-A) for the cases. The immunopositive results for some ER, PR and GCDFP-15 support the specimen diagnosis of metastasis from breast cancer, although certain lung cancers are known to be immunopositive for these antibodies (6-8). TTF-1 and SP-A are known to be specific markers for primary lung cancer, especially adenocarcinoma $(9,10)$. ER and PR staining was analyzed based on the Allred system, where intensity of staining is recorded on a 5 -scale grade $(0$, no staining; $1,20 \%$; $2,40 \% ; 3,60 \% ; 4,80 \%$; and 5, 100\%) (11). For GCDFP-15, TTF-1 and SP-A staining, tissue were positive if $\geq 10 \%$ of the tumor cells were stained. The final diagnosis of SPNs was confirmed based on the results of the immunohistochemical and morphopathological examinations.
Table I. Patient characteristics.

\begin{tabular}{|c|c|c|}
\hline Characteristics & $\mathrm{N}$ & $\%$ \\
\hline No. of patients & 30 & \\
\hline \multicolumn{3}{|l|}{ Age (years) } \\
\hline Mean & 58 & \\
\hline Range & $41-80$ & \\
\hline \multicolumn{3}{|c|}{ Nodule diameter (mm) } \\
\hline Mean & 12 & \\
\hline Range & $6-22$ & \\
\hline \multicolumn{3}{|l|}{ Nodule type } \\
\hline Solid & 14 & 47 \\
\hline Mixed GGO & 14 & 47 \\
\hline Pure GGO & 2 & 6 \\
\hline \multicolumn{3}{|l|}{ Coincidence } \\
\hline Yes & 7 & 23 \\
\hline No & 23 & 77 \\
\hline \multicolumn{3}{|l|}{ DFI (months) } \\
\hline Mean & 68 & \\
\hline Range & $6-229$ & \\
\hline \multicolumn{3}{|l|}{ Smoking status } \\
\hline Smoker & 3 & 10 \\
\hline Non-smoker & 27 & 90 \\
\hline \multicolumn{3}{|l|}{ Stage of breast cancer } \\
\hline \multicolumn{3}{|l|}{$\mathrm{pT}$} \\
\hline pT1 & 10 & 33 \\
\hline pT2 & 17 & 57 \\
\hline pT3 & 3 & 10 \\
\hline \multicolumn{3}{|l|}{$\mathrm{pN}$} \\
\hline pNO & 15 & 50 \\
\hline $\mathrm{pN} 1$ & 14 & 47 \\
\hline $\mathrm{pN} 2$ & 1 & 3 \\
\hline \multicolumn{3}{|c|}{ Laterality between breast cancer and SPN } \\
\hline Ipsilateral & 15 & 50 \\
\hline Contralateral & 15 & 50 \\
\hline \multicolumn{3}{|c|}{ Past history of malignancy } \\
\hline Breast cancer only & 27 & 90 \\
\hline \multicolumn{3}{|c|}{ Breast cancer and other types } \\
\hline Uterine cancer & 2 & 7 \\
\hline Colon cancer & 1 & 3 \\
\hline
\end{tabular}

GGO, ground-glass opacity; DFI, disease-free interval; SPN, solitary pulmonary nodule.

Data management. The in- and outpatient medical records were reviewed for the patients. The follow-up of the post-operative clinical course was conducted relying on outpatient medical records and telephone or written inquiries. The patients were retrospectively analyzed for age, diameter of SPN, disease-free interval (DFI), pre-operative followup period, smoking status, stage of breast cancer, laterality between breast cancer and SPN, past history of malignancy, 
Table II. Radiological features of solitary pulmonary nodules.

\begin{tabular}{|c|c|c|c|c|c|c|}
\hline \multirow[b]{2}{*}{ Diagnosis } & \multicolumn{2}{|c|}{ Pure GGO } & \multicolumn{2}{|c|}{ Mixed GGO } & \multicolumn{2}{|c|}{ Solid } \\
\hline & $\mathrm{N}$ & $\%$ & $\mathrm{~N}$ & $\%$ & $\mathrm{~N}$ & $\%$ \\
\hline Primary lung cancer & 2 & 6 & 11 & 37 & 7 & 24 \\
\hline \multicolumn{7}{|l|}{ Metastasis } \\
\hline Breast cancer & 0 & 0 & 3 & 10 & 4 & 13 \\
\hline Colon cancer & 0 & 0 & 0 & 0 & 1 & 3 \\
\hline Benign tumor & 0 & 0 & 0 & 0 & 2 & 7 \\
\hline Total & 2 & 6 & 14 & 47 & 14 & 47 \\
\hline
\end{tabular}

GGO, ground-glass opacity.
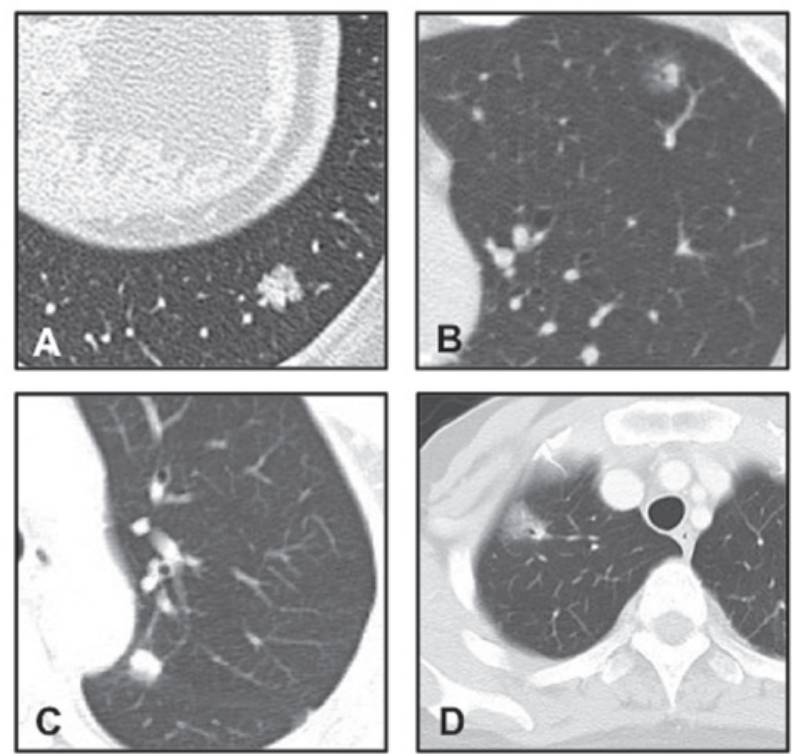

Figure 1. High-resolution CT images of SPNs are shown. Primary lung adenocarcinoma was classified as (A) solid and (B) mixed GGO. Metastasis from breast cancer was classified as (C) solid and (D) mixed GGO. CT, computed tomography; SPNs, solitary pulmonary nodules; GGO, ground-glass opacity.

histological subtype of the SPN, relapse and follow-up. The DFI was measured between the dates of initial treatment for breast cancer and the first SPN detection. The pre-operative follow-up period was measured between the dates of the first SPN detection and the date of operation. Overall survival (OS) was defined as the time period between the resection of the SPN and the date of the last follow-up or decease. Diseasefree survival (DFS) was defined as the time period between the resection of the SPN and the date of the first documented relapse or the last follow-up. The available survival data were updated in December, 2011.

Statistical analysis. OS and DFS were estimated using the Kaplan-Meier method. The Student's t-test and the $\chi^{2}$ test were used to compare percentages and the mean values, respectively. Seven patients with a pulmonary nodule detected simultaneously with breast cancer were excluded from the DFI analysis. The values were considered to indicate a statistically
Table III. Histological diagnosis of solitary pulmonary nodules in breast cancer patients.

\begin{tabular}{lcr}
\hline Diagnosis & No. of patients & $\%$ \\
\hline Malignancy & 28 & 93 \\
Primary lung cancer & 20 & 67 \\
Histology & & \\
Adenocarcinoma & 19 & 64 \\
Carcinoid & 1 & 3 \\
Pathological stage & & \\
IA (T1aN0M0) & 15 & 75 \\
IIA (T2aN1M0) & 1 & 5 \\
IIIA (T1aN2M0) & 3 & 5 \\
IV (T1aN0M1a) & 1 & 26 \\
Metastatic carcinoma & 8 & 23 \\
From breast cancer & 7 & 3 \\
From colon cancer & 1 & 7 \\
Benign condition & 2 & \\
Histology & & \\
Epithelioid cell granuloma & 1 & \\
Fibrosis and elastosis & 1 & \\
\hline
\end{tabular}

significant difference at $\mathrm{P}<0.05$. The statistical analyses were performed using the SPSS for Windows ver. 17.0 (SPSS Inc., Chicago, IL, USA).

\section{Results}

Patient groups. A differential diagnosis using only radiological features was difficult to make, since certain metastatic lung cancers revealed mixed ground-glass opacity (GGO) on the CT scans (Fig. 1 and Table II).

Of the 30 patients undergoing surgery during the study period, three underwent pre-operative CT-NAB yielding a diagnosis of primary lung adenocarcinoma. Twenty-six patients underwent wedge resections, while one required segmentectomy using VATS for the diagnosis of a deep intraparenchymal nodule. Definitive pathological diagnoses of 
Table IV. Immunohistochemical features of solitary pulmonary nodules.

\begin{tabular}{|c|c|c|c|c|c|c|c|c|c|c|c|}
\hline \multirow[b]{2}{*}{ Diagnosis } & \multirow[b]{2}{*}{ Total } & \multicolumn{2}{|c|}{ ER } & \multicolumn{2}{|c|}{ PR } & \multicolumn{2}{|c|}{ GCDFP-15 } & \multicolumn{2}{|c|}{ TTF-1 } & \multicolumn{2}{|c|}{ SPA } \\
\hline & & $\mathrm{N}$ & $\%$ & $\mathrm{~N}$ & $\%$ & $\mathrm{~N}$ & $\%$ & $\mathrm{~N}$ & $\%$ & $\mathrm{~N}$ & $\%$ \\
\hline Primary lung cancer & 19 & 3 & 16 & 0 & 0 & 3 & 16 & 15 & 79 & 13 & 68 \\
\hline \multicolumn{12}{|l|}{ Metastasis } \\
\hline Breast cancer & 7 & 4 & 57 & 2 & 29 & 5 & 71 & 0 & 0 & 2 & 29 \\
\hline Colon cancer & 1 & 0 & 0 & 0 & 0 & 0 & 0 & 0 & 0 & 0 & 0 \\
\hline Benign tumor & 2 & 0 & 0 & 0 & 0 & 0 & 0 & 0 & 0 & 0 & 0 \\
\hline
\end{tabular}

ER, estrogen receptor; PR, progesterone receptor; GCDFP-15, gross cystic disease fluid protein-15; TTF-1, thyroid transcription factor-1; SP-A, surfactant apoprotein-A.

Table V. Comparison of patient characteristics between primary lung cancer and metastasis from breast cancer.

\begin{tabular}{|c|c|c|c|c|c|c|c|}
\hline \multirow[b]{2}{*}{ Patient characteristics } & \multicolumn{3}{|c|}{ Primary lung cancer } & \multicolumn{3}{|c|}{ Metastasis from breast cancer } & \multirow[b]{2}{*}{ P-value } \\
\hline & $\mathrm{N}$ & Range & $\%$ & $\mathrm{~N}$ & Range & $\%$ & \\
\hline No. of patients & 20 & & & 7 & & & \\
\hline Age (years) & 62 & $41-77$ & & 54 & $41-58$ & & 0.121 \\
\hline Nodule diameter (mm) & 13 & $7-22$ & & 10 & $6-22$ & & 0.237 \\
\hline DFI (months) & 94 & $6-229$ & & 41 & $12-88$ & & 0.031 \\
\hline $\begin{array}{l}\text { Pre-operative follow-up period } \\
\text { (months) }\end{array}$ & 11 & $1-84$ & & 5 & $1-12$ & & 0.232 \\
\hline Smoker & 1 & & 5 & 1 & & 14 & 0.459 \\
\hline Ipsilaterality & 10 & & 50 & 4 & & 57 & 0.765 \\
\hline \multicolumn{8}{|l|}{ Stage of breast cancer } \\
\hline \multicolumn{8}{|l|}{$\mathrm{T}$} \\
\hline $\mathrm{T} 1,2$ & 18 & & 90 & 6 & & 86 & 0.610 \\
\hline $\mathrm{T} 3$ & 2 & & 10 & 1 & & 14 & \\
\hline \multicolumn{8}{|l|}{$\mathrm{N}$} \\
\hline N0 & 10 & & 50 & 2 & & 29 & 0.298 \\
\hline $\mathrm{N} 1,2$ & 10 & & 50 & 5 & & 71 & \\
\hline
\end{tabular}

DFI, disease-free interval.

SPN are listed in Table III, and immunohistochemical features are shown in Table IV. Three patients, who underwent only wedge resection by VATS due to small nodules showing pure or mixed GGO, were diagnosed with non-invasive primary lung cancer. In two cases, the absence of malignant cells was demonstrated by intraoperative examination, thus the operation was terminated (one patient received a wedge resection and one segmentectomy). Intraoperative diagnoses of malignant lesions were made in 22 patients by wedge resection, while 12 patients were diagnosed with primary lung cancer, 11 of whom had adenocarcinoma and one a carcinoid tumor. Ten of these 11 patients underwent lobectomy and lymph node dissection, whereas one underwent segmentectomy and lymph node dissection. In one case, dissemination was found intraoperatively and the operation was terminated subsequent to the wedge resection and the dissection of the dissemination. In five cases, intraoperative examination did not distinguish between primary or metastatic adenocarcinoma. These patients underwent lobectomy or segmentectomy and lymph node dissection. Two were definitively diagnosed with primary lung cancer and three with metastases of breast cancer. Five cases were diagnosed as metastatic adenocarcinoma, and these operations were terminated after wedge resection. Four of these patients had metastases from breast and colon cancers. Eight patients diagnosed with metastatic lung cancer started systemic treatment subsequent to pulmonary resection.

Of the 20 patients with primary lung cancer, 15 (75\%) had stage IA tumors (T1aN0M0), one a stage IIA tumor (T2aN1M0), three stage IIIA tumors (T1aN2M0) and one a stage IV tumor (T1aN0M1a), based on the International Association for the 
A

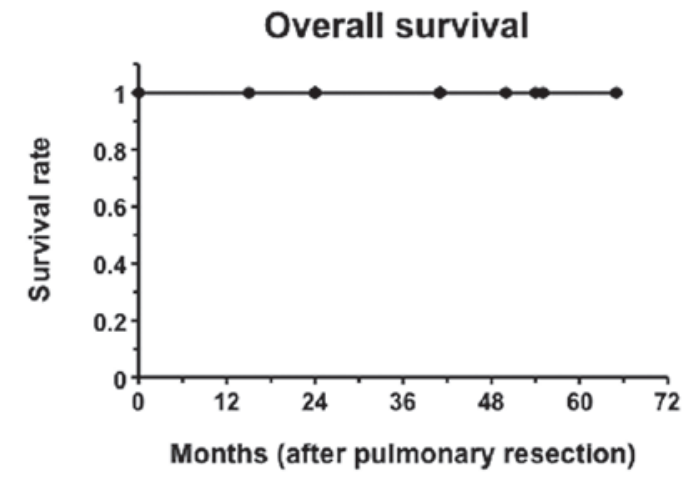

B

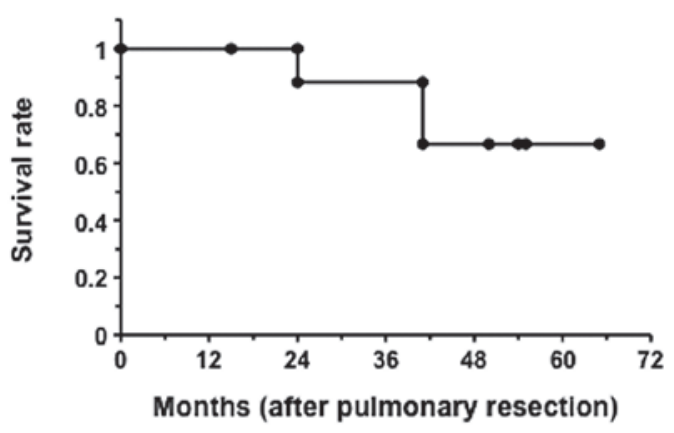

Figure 2. Kaplan-Meier survival plots after pulmonary resection for patients diagnosed with pulmonary metastasis from breast cancer $(\mathrm{n}=7)$ are shown.

A

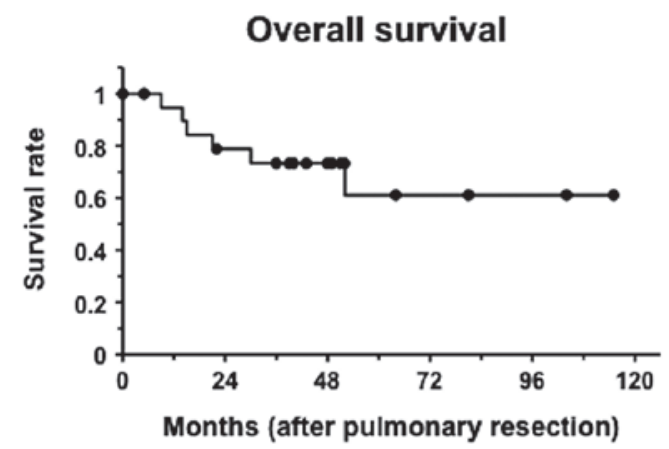

B

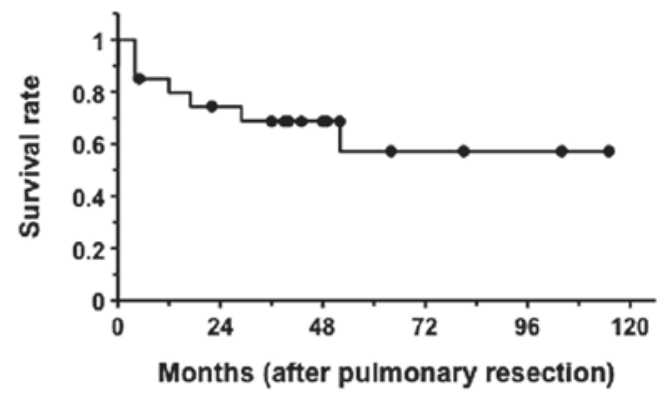

Figure 3. Kaplan-Meier survival plots after pulmonary resection for patients diagnosed with primary lung cancer (n=20) are shown.

Study of Lung Cancer (IASLC) criteria (7th edition) (Aurora, CO, USA) (Table III). The median follow-up period between the first SPN detection and the pulmonary resection was 9.5 months, while the follow-up period did not exceed six months in 12 of the 15 stage IA primary lung cancer patients.

A significant difference was observed only in the DFI between the primary lung cancer and metastasis from breast cancer (Table V). The average DFI in patients with primary lung cancer and metastatic breast cancer was 94 (range, 6-229) months and 41 (range, 12-88) months, respectively. DFI of the primary lung cancer group was markedly longer compared to the metastatic breast cancer group $(\mathrm{P}=0.031)$. However, in 2 of 7 patients with metastases from breast cancer, DFI was $>5$ years. In 6 of the 20 patients with primary lung cancer, DFI was $<5$ years, while in seven patients, pulmonary nodules were detected during the pre-operative examination of breast cancer.

Survival and outcomes. Follow-up was complete for the patients in this study. The median follow-up was 46 months (range, 5-115). The five-year survival rate for lung metastasis from breast cancer patients was $100 \%$ subsequent to pulmonary resection, although the median follow-up was 52 months (Fig. 2). Of these 7 patients, five were event-free and two had breast cancer relapse: one with lung metastasis and the other with hilar lymph nodes. For the patient with lung metastasis relapse, subsequent lung resection was performed, with no recurring relapse.
The five-year survival period for the 20 primary lung cancer patients was $61.1 \%$ subsequent to pulmonary resection (Fig. 3). Six patients diagnosed with primary lung cancer succumbed to the disease during the observation period, and four of lung cancer relapse (three had stage IIIA tumors and one a stage IV tumor), one from breast cancer relapse and one from cerebral apoplexy. The remaining 14 patients, with stage I or IIA tumor, were event-free, with the exception of one patient, who developed a new primary lung cancer.

\section{Discussion}

The present study demonstrated that $93 \%$ of SPNs found in patients with breast cancer were malignant. The majority of pathological diagnosis of these SPNs was primary lung cancer (67\%) or pulmonary metastasis from breast cancer (23\%). The early resection of SPNs was demonstrated to possibly result in good prognosis in patients with malignant tumors, regardless of their diagnosis as primary lung cancer or metastasis.

A differential diagnosis between primary and metastatic lung cancer using radiological features was difficult, as seen in previous studies $(5,6)$. No visible differences were detected in the radiological features or the dimension (Fig. 1; Table II). GGO is one of the characteristic signs of primary lung adenocarcinoma, although cases of metastatic lung tumor from the adenocarcinoma of the gastrointestinal tract or malignant melanoma showing GGOs have also been reported $(12,13)$. In the present study, $>40 \%$ (3/7) of the metastases from breast 
cancer showed mixed GGO on CT. Therefore, an SPN showing GGO on CT is not likely to indicate pulmonary adenocarcinoma in patients with a history of breast cancer. Furthermore, a differential diagnosis of focal GGO includes inflammatory diseases, focal fibrosis and atypical adenomatous hyperplasia (14). Several studies have demonstrated that sometimes even a benign and malignant distinction is difficult to make $(5,6)$. Therefore, a qualitative diagnosis is required for the definitive diagnosis of SPNs in breast cancer patients.

Previous studies have reported that $\sim 50 \%$ of SPNs are benign $(2,15)$. However, when the population is limited to patients, who have undergone surgery for breast cancer, the malignancy rate of SPNs has been shown to be $>80 \%(5,6,16)$. Thus, it is necessary to monitor SPNs in patients with breast cancer as potentially malignant, as in primary or metastatic lung cancers. In the present study, 93\% of SPNs found in patients with breast cancer were malignant, while $67 \%$ were diagnosed as primary lung cancer. The percentage of primary lung cancer was the highest in previous studies $(5,6)$, as in their case of Rena et al (5), who reported that $82 \%$ of the SPNs found in patients subsequent to mastectomy were malignant, while $48 \%$ were diagnosed as primary lung cancer. Consistent with the present findings, that study reported that the major population of SPNs found in patients subsequent to mastectomies was primary lung cancer, however, the frequency of primary lung cancer in this study was higher, especially in adenocarcinoma (19/20 patients with primary lung cancer). This tendency may be associated with the increasing number of patients with lung adenocarcinoma, especially among young Asian, non-smoking women $(17,18)$; all patients but three were non-smoking Japanese women. Okasaka et al (6) reported that $100 \%$ (48/48) of their cases were malignant, while $83 \%$ were diagnosed as breast cancer metastases. In that study, specimens were obtained by CT-NAB or transbronchial lung biopsy (TBLB) in $44(91.7 \%)$, and by surgical resection in 4 patients. Moreover, 32 patients were diagnosed by conventional pathological examination, and immunohistochemical staining and molecular marker examination were performed for the remaining patients in a step-wise manner (6). By contrast, in this study, the entire tumor was evaluated by surgical resection and immunohistochemical staining performed in all cases. The discrepancy in the rate is likely to be due to the different specimen collection method or candidates for immunohistochemical staining.

Lung resection is a controversial treatment for pulmonary metastasis from breast cancer $(5,19-23)$. In this study, the five-year survival period for solitary lung metastasis from breast cancer patients was $100 \%$ after pulmonary resection. The majority of breast oncologists opt for systemic treatment, even in the case of a single lesion metastasis, given that pulmonary metastasis is a systemic disease (5). The median survival period of pulmonary metastasis patients subsequent to systemic treatment remains approximately 24 months $(19,20)$. Several studies have suggested that surgical treatment for operable lung metastases from breast cancer followed by systemic treatment prolongs survival to a greater extent compared to standard systemic treatment alone, in patients with $<4$ pulmonary metastases or with a DFI $>3$ years $(21-23)$. The present study also suggests that pulmonary resection for solitary lung metastasis from breast cancer is likely to contribute to the improvement of prognosis, although a combined systemic therapy might be required.

Furthermore, a substantial discordance between the human epidermal growth factor receptor 2 (HER2) expression and hormone receptors, such as ER and PR, in primary and recurrent tumors in patients with recurrent breast cancer has been reported $(24,25)$. These reports showed a $\sim 40 \%$ discordance in the hormone receptor status and 8-14\% discordance in HER2 status $(24,25)$. Discordant cases have poor survival rates, probably due to inappropriately targeted therapies. In the present study, discordance in the PR status was also observed, although in only one patient (14.3\%). Therefore, tissue confirmation is suggested for the appropriate treatment in patients with clinically or radiologically suspected metastasis from breast cancer. Moreover, the entire tumor needs to be examined to avoid intratumoral heterogeneity generating discordance in a limited number of specimens $(25,26)$.

To provide an appropriate treatment, the diagnosis of an SPN must be early and certain. Consistent with the findings of this study, DFI for metastases from breast cancer has been demonstrated to be significantly shorter compared to primary lung cancer (5). However, $29 \%$ of patients with metastases from breast cancer have DFI $>5$ years, while $30 \%$ of primary lung cancer cases have DFI $<5$ years. Although the DFI is available for assistance with differential diagnosis, it is difficult to diagnose only by clinical data, including the DFI criteria. Therefore, a pathological examination is necessary to confirm the diagnosis. Step-wise examination using biopsy specimens, initiating with CT-NAB or TBLB, for the differential diagnosis of SPN $(6,27)$ has been previously made. However, limitations and complications occur during these examinations. The examinations are limited due to the tumor location and size, since when the nodules are small or in an intricate location, it is difficult to extract an appropriate specimen for pathological examination, including several immunohistochemical stainings $(2,28)$. Another limitation of these examinations is their being only diagnostic at best, rather than therapeutic, albeit being less invasive compared to surgery (2). Moreover, several complications, such as pneumothorax, intrapulmonary hemorrhage, air embolism and pleural implantation, associated with CT-NAB, have been reported (29). Being a minimally invasive surgical procedure with high diagnostic sensitivity and specificity, VATS has become a popular technique for nodule resection (30). Additionally, VATS may be converted to therapeutic resection when a nodule is diagnosed intraoperatively as primary lung cancer.

Evidently, the earlier the stage of primary lung cancer, the better the prognosis. In primary lung cancer cases in this study, $75 \%$ of the patients had a stage IA tumor at the time of the surgery. The patients with stage IA tumor are still alive, with the exception of two patients, deceased from other causes. The five-year cancer-specific survival period for stage IA primary lung cancer patients was $100 \%$ subsequent to pulmonary resection, possibly due to a relatively short pre-operative follow-up period leading to an earlier detection and more frequent opportunity for chest CT scan compared to cancer-free women. Advanced-stage lung cancer patients had a tendency for long follow-up or delay in the detection of pulmonary involvement since the DFI occurred over a long period of time and no periodic follow-up was carried 
out. Therefore, early resection of SPN found in patients with breast cancer is likely to also contribute to the improvement of prognosis for primary lung cancer patients.

In conclusion, this study has shown that SPNs found in patients with breast cancer have a high probability of malignancy, especially primary lung adenocarcinoma. Furthermore, the early resection of SPN led to good prognosis in patients diagnosed with both primary and metastatic lung cancers. Thus, early pathological diagnosis by surgical resection was suggested to be conducted for the early diagnosis and the appropriate treatment of SPNs in patients with breast cancer.

\section{References}

1. Jemal A, Siegel R, Ward E, Hao Y, Xu J and Thun MJ: Cancer statistics. CA Cancer J Clin 59: 225-249, 2009.

2. Tan BB, Flaherty KR, Kazerooni EA and Iannettoni MD: The solitary pulmonary nodule. Chest 123: 89-96, 2003.

3. Jeong YJ, Yi CA and Lee KS: Solitary pulmonary nodules: detection, characterization, and guidance for further diagnostic workup and treatment. Am J Roentgenol 188: 57-68, 2007.

4. Lorigan P, Califano R, Finn CF, Howell A and Thatcher N: Lung cancer after treatment for breast cancer. Lancet Oncol 11: $1184-1192,2010$

5. Rena O, Papalia E, Ruffini E, Filosso PL, Oliaro A, Maggi G and Casadio C: The role of surgery in the management of solitary pulmonary nodule in breast cancer patients. Eur J Surg Oncol 33 546-550, 2007.

6. Okasaka T, Usami N, Mitsudomi T, Yatabe Y, Matsuo K and Yokoi K: Stepwise examination for differential diagnosis of primary lung cancer and breast cancer relapse presenting as a solitary pulmonary nodule in patients after mastectomy. J Surg Oncol 98: 510-514, 2008.

7. Mazoujian G, Pinkus GS, Davis S and Haagensen DE Jr: Immunohistochemistry of a gross cystic disease fluid protein (GCDFP-15) of the breast. A marker of apocrine epithelium and breast carcinomas with apocrine features. Am J Pathol 110: 105-112, 1983.

8. Yang M and Nonaka D: A study of immunohistochemical differential expression in pulmonary and mammary carcinomas. Mod Pathol 23: 654-661, 2010.

9. Holzinger A, Dingle S, Bejarano PA, Miller MA, Weaver TE, DiLauro R and Whitsett JA: Monoclonal antibody to thyroid transcription factor-1: production, characterization, and usefulness in tumor diagnosis. Hybridoma 15: 49-53, 1996.

10. Nicholson AG, McCormick CJ, Shimosato Y, Butcher DN and Sheppard MN: The value of PE-10, a monoclonal antibody against pulmonary surfactant, in distinguishing primary and metastatic lung tumours. Histopathology 27: 57-60, 1995.

11. Allred DC, Harvey JM, Berardo M and Clark GM: Prognostic and predictive factors in breast cancer by immunohistochemical analysis. Mod Pathol 11: 155-168, 1998.

12. John HW and Benedek B: CT halo sign in pulmonary metastases from mucinous adenocarcinoma of the pancreas. South Med J 94: 448-449, 2001.

13. Okita R, Yamashita M, Nakata M, Teramoto N, Bessho A and Mogami H: Multiple ground-grass opacity in metastasis of malignant melanoma diagnosed by lung biopsy. Ann Thorac Surg 79: e1-e2, 2005

14. Yanagitani N, Kaira K, Ishizuka T, Aoki H, Utsugi M, Shimizu Y, Sugano N, Endou K, Hisada T and Mori M: Multiple lung metastases presenting as ground-glass opacities in a pulmonary adenocarcinoma: a case report. Cases J 2: 6910, 2009.

15. Swanson SJ, Jaklitsch MT, Mentzer SJ, Bueno R, Lukanich JM and Sugarbaker DJ: Management of solitary pulmonary nodule: role of thoracoscopy in diagnosis and therapy. Chest 116 (Suppl 6): S523-S524, 1999.
16. Casey JJ, Stempel BG, Scanlon EF and Fry WA: The solitary pulmonary nodule in the patient with breast cancer. Surgery 4 : 801-805, 1984

17. Charloux A, Quoix E, Wolkove N, Small D, Pauli G and Kreisman H: The increasing incidence of lung adenocarcinoma: reality or artifact? A review of the epidemiology of lung adenocarcinoma. Int J Epidemiol 26: 14-23, 1997.

18. Lam B, Lam WK, Lam CL, Ooi GC, Ho JCM, Wong MP and Tsang KW: Adenocarcinoma of the lung in Chinese patients: a revisit and some perspectives from the literature. Postgrad Med J 77: 708-712, 2001.

19. Greenberg PA, Hortobagyi GN, Smith TL, Ziegler LD, Frye DK and Buzdar AU: Long-term follow-up of patients with complete remission following combination chemotherapy for metastatic breast cancer. J Clin Oncol 14: 2197-2205, 1996.

20. Diaz-Canton EA, Valero V, Rahman Z, Rodriguez-Monge E, Frye D, Smith T, Buzdar AU and Hortobagyi GN: Clinical course of breast cancer patients with metastases confined to the lungs treated with chemotherapy. The University of Texas M.D. Anderson Cancer Center experience and review of the literature. Ann Oncol 9: 413-418, 1998.

21. Yhim HY, Han SW, Oh DY, Han W, Im SA, Kim TY, Kim YT, Noh DY, Chie EK, Ha SW, et al: Prognostic factors for recurrent breast cancer patients with an isolated, limited number of lung metastases and implications for pulmonary metastasectomy. Cancer 116: 2890-2901, 2010.

22. Chen F, Fujinaga T, Sato K, Sonobe M, Shoji T, Sakai H, Miyahara R, Bando T, Okubo K, Hirata T, et al: Clinical features of surgical resection for pulmonary metastasis from breast cancer. Eur J Surg Oncol 35: 393-397, 2009.

23. Yoshimoto M, Tada K, Nishimura S, Makita M, Iwase T, Kasumi F, Okumura S, Sato Y and Nakagawa K: Favourable long-term results after surgical removal of lung metastases of breast cancer. Breast Cancer Res Treat 110: 485-491, 2008.

24. Simmons C, Miller N, Geddie W, Gianfelice D, Oldfield M, Dranitsaris G and Clemons MJ: Does confirmatory tumor biopsy alter the management of breast cancer patients with distant metastases? Ann Oncol 20: 1499-1504, 2009.

25. Liedtke C, Broglio K, Moulder S, Hsu L, Kau SW, Symmans WF, Albarracin C, Meric-Bernstam F, Woodward W, Theriault RL, et al: Prognostic impact of discordance between triple-receptor measurements in primary and recurrent breast cancer. Ann Oncol 20: 1953-1958, 2009.

26. Gong Y, Symmans WF, Krishnamurthy S, et al: Optimal fixation conditions for immunohistochemical analysis of estrogen receptor in cytologic specimens of breast carcinoma. Cancer 102: 34-40, 2004.

27. Gould MK, Fletcher J, Iannettoni MD, Lynch WR, Midthun DE, Naidich DP and Ost DE: Evaluation of patients with pulmonary nodules: when is it lung cancer?: ACCP evidence-based clinical practice guidelines (2nd edition). Chest 132 (Suppl 3): S108-S130, 2007.

28. Li H, Boiselle PM, Shepard JO, Trotman-Dickenson B and McLoud TC: Diagnostic accuracy and safety of CT-guided percutaneous needle aspiration biopsy of the lung: comparison of small and large pulmonary nodules. AJR Am J Roentgenol 167: 105-109, 1996.

29. Inoue M, Honda O, Tomiyama N, Minami M, Sawabata N, Kadota Y, Shintani Y, Ohno Y and Okumura M: Risk of pleural recurrence after computed tomographic-guided percutaneous needle biopsy in stage I lung cancer patients. Ann Thorac Surg 91: 1066-1072, 2011.

30. McCormack PM, Bains MS, Begg CB, Burt ME, Downey RJ, Panicek DM, Rusch VW, Zakowski M and Ginsberg RJ: Role of video-assisted thoracic surgery in the treatment of pulmonary metastases: results of a prospective trial. Ann Thorac Surg 62: 213-217, 1996. 